\title{
МЕТОДИКА МЕДИКО-ЭКОНОМИЧЕСКОЙ ОЦЕНКИ РЕСУРСНОГО ПОТЕНЦИАЛА МЕДИЦИНСКИХ ОРГАНИЗАЦИЙ
}

\author{
(c) 2021 Качкова Ольга Евгеньевна \\ кандидат экономических наук, доцент, \\ заместитель руководителя Департамента аудита и корпоративной отчетности \\ Финансовый университет при Правительстве Российской Федерации, Россия, Москва \\ https://orcid.org/0000-0003-1439-2845
}

(c) 2021 Кришталева Таисия Ивановна

доктор экономических наук, профессор Департамента аудита и корпоративной отчетности Финансовый университет при Правительстве Российской Федерации, Россия, Москва https://orcid.org/0000-0003-4297-5917

В статье показана оценка эффективности ресурсных затрат и конкурентоспособности медицинских организаций, сравнение больниц по эффективности, доступности и качеству медицинской помощи, представлены результаты исследований влияния современных информационнотехнологических ресурсов на другие смежные показатели эффективности функционирования больничных учреждений, поиска причинно-следственных связей между отдельными показателями и объяснения причин с точки зрения рассмотрения процессов, происходящих в медицинских организациях, и их совершенствования. Оценка причинно-следственных связей неэффективного или низкоэффективного использования ресурсов здравоохранения в медицинских организациях и поиск резервов для совершенствования процессов оказания медицинской помощи должна осуществляться комплексно с использованием дополняющих друг друга нормативного, статистического и аналитического методов планирования ресурсного обеспечения.

Ключевые слова: эффективность использования ресурсов; качество медицинской помощи; длительность лечения; телемедицинские технологии; медицинское оборудование; бережливые технологии; производственный потенциал.

Оценке эффективности ресурсных затрат и конкурентоспособности медицинских организаций в зависимости от их организационных правовых форм посвящено исследование американских ученых, сравнивших небольшие больницы, специализирующиеся на кардиологических, ортопедических или хирургических услугах (SSHs) с многопрофильными общественными больницами [1]. Сторонники SSH утверждают, что эти организации могут установить новый конкурентный критерий для больничных услуг, способствуя эффективности затрат, расширяя выбор пациентов и предоставляя качественную медицинскую помощь по конкурентоспособным ценам. Противники SSH утверждают, что эти организации порождают недобросовестную конкуренцию, ориентируясь на направления пациентов, предлагая услуги, которые способствуют чрезмерному использованию, сосредотачиваясь на наиболее прибыльных пациентах, и ограничивая способность многопрофильных общественных больниц с полным спектром ус- луг перекрестно субсидировать убыточные услуги. Помимо вопроса о том, кто контролирует ресурсы больницы, возникает вопрос о том, кто владеет этими ресурсами. Общей характеристикой SSH является то, что это некоммерческая собственность врачей.

Стимулы владельцев-врачей потенциально противоречивы. С одной стороны, они напрямую выигрывают от увеличения прибыли больниц и более высокой степени экономической эффективности способами и стимулами, отличающимися от существующих в больницах общего профиля.

С другой стороны, эти владельцы-врачи также получают непосредственную выгоду от более высокой производительности в своей клинической практике за счет более высоких платежей. Некоторые из их действий, направленных на повышение клинической эффективности, могут нанести ущерб эффективности затрат в больницах, таких как планирование операционной, способствующее эффективности и удобству хи- 
рурга, а не минимизировать операционные расходы больницы.

Результаты исследования показали, что более низкие затраты при использовании ресурсов отмечены в коммерческих больницах. Расслоение показателей неэффективности среди больниц с полным спектром услуг также показывает меньшую неэффективность в коммерческих больницах, поэтому среди больниц с полным спектром услуг на изучаемых рынках, стимул для получения прибыли, по-видимому, способствует повышению эффективности, связанной с затратами.

В систематическом обзоре, отражающем данные о результатах исследований, проведенных в странах Европейского Союза - Италии, Германии, Франции, Греции, Австрии, Испании и Португалии, утверждается, что государственные больницы являются, по крайней мере, такими же эффективными или более эффективными, чем частные больницы [2].

Сравнение больниц проводилось по трем критериям - эффективность, доступность и качество медицинской помощи. Под термином «эффективность» авторы понимали степень достижения цели по отношению к потребленным ресурсам. Эффективность отражается в нескольких показателях, таких как техническая эффективность (максимальный выход из заданного набора входов или минимальный набор входов с заданным набором выходов), экономическая эффективность (учет технической эффективности для начальной цены), масштабная эффективность (когда размер единицы является оптимальной) и / или эффективность распределения / прибыли (минимизация затрат или максимизация прибыли). Доступность делится на финансовую доступность, физический доступ, информированность и своевременный доступ (например, время ожидания).

Качество медицинской помощи структурировано в соответствии с донабедиановской (Donabedian) моделью структуры, процесса и результатов. Модель Донабедяна продолжает оставаться доминирующей парадигмой для оценки качества медицинской помощи.

Некоторые изученные показатели, такие как длительность лечения, были классифицированы и отнесены к различным критериям и показали свою неоднородность как в части отдельных заболеваний, так и применительно к больницам разных форм собственности, в том числе внутри одной группы.

Оценка эффективности использования ресурсов здравоохранения по длительности лечения признана недостоверной в исследовании, проведенном в медицинских центрах США в период с 2007 по 2011 г. [3]. По мнению исследователей оценка эффекта лечения по данному показателю не только не надежна и не полезна, но и нет доказательств того, что можно руководствоваться этим выбором. Чтобы получить результаты, которые минимизируют проблемы и максимизируют достоверность, исследователи должны сопоставлять и анализировать группы лечения и сравнения только по базовым факторам. Авторами предлагается использовать временной интервенционный рейтинг, который может дать результаты, являющиеся более надежными и полезными, чем результаты, полученные с использованием показателя «длительность лечения» [4]. Исследователи из Южной Кореи, наоборот, считают показатель «длительность лечения» важнейшим показателем эффективности деятельности больницы. Сокращение длительности лечения снижает риск инфицирования и побочных эффектов от лекарств, улучшает качество лечения и обеспечивает увеличение прибыли в стационаре [5]. Цель их исследования состояла в том, чтобы определить, какие факторы связаны с продолжительностью пребывания в больнице, на основе электронных медицинских карт, для более эффективного управления ресурсами медицинских организаций и пребыванием в больнице. По мнению исследователей продолжительность пребывания в больнице зависит от времени перевода пациентов между отделениями, времени задержки выписки пациента, частоты хирургических вмешательств, частоты диагностики, тяжести пациента, типа страхования.

Авторы пришли к выводу, что исследование продолжительности пребывания в стационаре помогает больницам более эффективно управлять своими ресурсами и пациентами, разрабатывать лучшие организационно-клинические алгоритмы ведения пациентов, полезные для стационарного лечения. Исходя из переменных, определенных в этом исследовании, может потребоваться улучшение финансовой структуры больниц и разработка институциональных подходов для снижения оплаты медицинских услуг пациентам путем содействия эффективному использованию ресурсов больницы и сокращения 
продолжительности пребывания в стационаре через систему, подлежащую постоянному мониторингу. Устранение ненужного пребывания в стационаре - это стратегия сокращения общенациональных медицинских расходов.

Несомненную ценность представляют результаты исследований влияния современных информационно-технологических ресурсов на другие смежные показатели эффективности функционирования больничных учреждений. Так, в исследовании больниц штата Флорида США в период с 2011 по 2016 годы изучалось влияние телемедицинских технологий на показатели летальности, продолжительности пребывания на койке и стоимости среди пациентов в отделениях прогрессивного (постепенного) ухода [4].

Результаты исследования показали, что телемедицинские услуги значительно снизили летальность в отделениях прогрессивного (постепенного) ухода несмотря на то, что пациенты были в старшей возрастной группе и имели тяжелые заболевания с высоким риском смерти. Увеличение продолжительности пребывания в стационаре после лечения и общих средних прямых затрат, включая расходы на телемедицину, совпало с улучшением показателей выживаемости. Телемедицинские услуги снизили общую смертность и продолжительность пребывания в отделениях прогрессивного ухода без существенных расходов.

Отдельные исследования, проводимые в отношении использования определенных ресурсов здравоохранения или медицинских организаций не дают, однако, возможности их комплексной сравнительной оценки в динамике и применительно к конкретным странам или регионам.

Данная информация доступна в ежегодной периодике международных организаций или служб, деятельность которых так или иначе сопряжена с системой здравоохранения.

Данные отчета стран Организации экономического сотрудничества и развития за 2017 год [6] представляют интерес с точки зрения оценки таких показателей, как, например, «время ожидания плановой операции», позволяющий косвенно оценить эффективность использования ресурсов здравоохранения и системы планирования оказания медицинской помощи в зависимости от заболевания, «обеспеченность населения койками», «доля коечного фонда в зависимости от функционального предназначения», «заполняемость экстренных (неотложных) коек», «средняя продолжительность пребывания в больнице» и некоторых других.

Оценка таких ресурсов здравоохранения, как «медицинское оборудование» приводится применительно к численности населения. Так, отмечается, что не существует общего руководства или эталона относительно идеального количества КТ-сканеров или единиц МРТ на популяцию.

Значительное число зарубежных исследований последних лет посвящено уже не столько сравнительной оценке использования ресурсов здравоохранения в динамике, сколько поиску причинно-следственных связей между отдельными показателями и объяснению причин с точки зрения рассмотрения процессов, происходящих в медицинских организациях, и их совершенствования. Так, ставшее трендом последних лет внедрение бережливых технологий, дает возможность оптимизации использования ресурсов здравоохранения путем воздействия на процессы, сопряженные с оказанием медицинской помощи, или непосредственно связанные с ней. Непосредственные результаты были также более значительными в больницах, в которых работники принимали активное участие в изменении рутинных процессов с применением бережливых технологий. Фактором, который значительно повлиял на результаты во второй и третий год постимплементации, был уровень постоянной приверженности руководства больниц бережливым технологиям. Эффективность внедрения бережливых технологий при должной приверженности и заинтересованности со стороны руководства больниц и медицинского персонала, формировании единой сплоченной команды также доказана в исследованиях по современным управленческим практикам в больницах США [7], Швеции [8], Великобритании [9].

Адекватному планированию ресурсов и оценке эффективности деятельности медицинской организации препятствует отсутствие единых критериев использования имущества медицинской организации, в т.ч. медицинского оборудования, отсутствие единых расчетных норм времени на проведение инструментальнодиагностических и лабораторных исследований. Для оценки причинно-следственных связей неэффективного или низкоэффективного использования ресурсов здравоохранения в ме- 
дицинских организациях и поиска резервов для совершенствования процессов оказания медицинской целесообразно провести анализ основных процессов с их оптимизацией, используя lean-технологии (устранение всех видов потерь).

При анализе ресурсных затрат медицинских организаций необходимо оценить последствия передачи отдельных административных и клинических услуг на аутсорсинг, сокращения неэффективных медицинских вмешательств, продолжительного пребывания пациентов в стационаре, использования телемедицинских технологий, внедрения систем мотивации и стимулов для медицинских работников к повышению производительности труда.

Исследование ресурсов медицинских организаций и разработка механизмов улучшения эффективности их использования, в том числе при анализе и оценке диагностических, лечебных и организационных процессов, остается актуальной задачей для организаторов и управленцев в сфере здравоохранения как в Российской Федерации, так и за рубежом.

Сферу интересов ученых представляют изучение использования следующих ресурсов: кадровых (КР) - обеспечение медицинским персоналом, совершенствование профессиональной квалификации, обучение современным лечебнодиагностическим навыкам и технологиям, повышение производительности труда, формирование мотивации и заинтересованности в объемах и качестве оказываемой медицинской помощи и др.; материально-технических (МТР) - обеспечение медицинским оборудованием (диагностическим и лечебным), медицинскими изделиями, лекарственными препаратами, коечным фондом, помещениями медицинского и немедицинского назначения, эффективность их использования по критериям технической и фактической производительности, соответствия нормативным требованиям и эксплуатационным характеристикам, соответствия стандартам и порядкам оказания медицинской помощи и др.; финансово-экономических (ФЭР) - распределение источников финансового обеспечения по статьям расходов, расчет нормативных и фактических единиц финансовых затрат на оказание медицинских услуг, качество планирования финансово-хозяйственной деятельности и работы с финансовой задолженностью, оценка рентабельности активов и др.; информационнокоммуникационных (ИКР) - влияние информа- ционных систем на принятие управленческих решений, отработка технологий моделирования процессов оказания медицинской помощи и расчет планируемых показателей экспериментального внедрения, влияние телемедицинских технологий на интерпретацию результатов диагностики и лечения и др.; организационноуправленческих (ОУР) - изучение возможности оптимизации оказания медицинской помощи и совершенствование логистики [10]; применение методик и технологий управления персоналом и создание мотивационных стимулов к повышению производительности труда и др.

При анализе по метрикам процессов, происходящих в медицинской организации, возможно ранжирование причин и целенаправленное воздействие на них в части повышения эффективности использования ресурсов. Таким образом, в целевой модели медико-экономической оценки ресурсного потенциала медицинских организаций, оказывающих медицинскую помощь в стационарных условиях, необходим комплексный подход и применение технологий систематического поиска и полного определения причин возникновения проблем с использованием ресурсов [11].

Оценку причинно-следственных связей неэффективного или низкоэффективного использования ресурсов здравоохранения в медицинских организациях и поиск резервов для совершенствования процессов оказания медицинской помощи целесообразно проводить в соответствии с принципами и подходами, используемыми в бережливом производстве [12].

Для анализа следует использовать статистический, нормативный и аналитический методы. При этом во всех случаях считается, что МО полностью обеспечена материальными (медицинское оборудование в исправном (работоспособном) состоянии, лекарственные препараты, консервированная кровь и ее компоненты, иные расходные материалы / материальные запасы) и финансовыми ресурсами.

При анализе внешних производственных связей организации будем рассматривать следующие классы процессов, субъектами которых являются российские юридические лица, агенты - представители пациента, а также сам гражданин - пациент, получающий медицинскую помощь (здесь и далее используются общепринятые трехсимвольные обозначения субъектных пар, где в качестве символа-связки исполь- 
зуется цифра '2'):

G2G - процессы взаимодействия между государственными органами $(G)$, участвующими в организации предоставления медицинских услуг пациенту;

G2B - процессы предоставления госорганами услуг бизнес-организациям (B), а также процессы осуществления контрольно-надзорной деятельности в отношении бизнес-организаций (интеракции «государство - бизнес»);

B2G - процессы подготовки и предоставления бизнес-организацией информации госорганам (интеракции «бизнес - государство»);

В2В - процессы взаимодействия между бизнес-организациями, когда один из субъектов является получателем и/или заказчиком, а другой - исполнителем работы и/или оказывает услуги (интеракции «бизнес - бизнес»); *

G2C - процессы предоставления государственным органом услуг непосредственно гражданам (C; интеракции «государство -гражданин»);

В2C - процессы выполнения бизнесорганизацией работ и/или предоставления услуг гражданам (интеракции «бизнес -гражданин»);

С2В - процессы оценки гражданами качества предоставляемых услуг и/или выполнения работ бизнес-организациями.

Для анализа внешних производственных связей организации будем использовать те же условные буквенные коды, которые использовали при анализе производственных связей между структурными подразделениями МО, учитывая также необходимость персонификации услуги и обработки персональных данных пациента (ПД), либо отсутствие необходимости в ее персонификации (НП) [13].

В настоящее время система SH сбалансирована не до конца, поскольку по ряду медицинских организаций наблюдаются кардинально противоположенные диспропорции. Для одних характерно WP < LP, что приводит к формированию длительного листа ожидания, провоцирующее выбытие пациентов в другие медицинские организации аналогичного профиля медицинской помощи.

Для других МO наоборот, VP < WP, означающее заблаговременное выполнение плановых объемов медицинской помощи, провоцирующее недозагруженность ресурсов МО и в конечном итоге неэффективное использование кадрового потенциала, медицинского оборудования, коечного фонда, площадей.

Таким образом, продолжительность ожидания СПС определяется дефицитом медицинских мощностей (WP < LP) и финансовых ресурсов (VP < WP), а также иными причинами, не связанными с этими дефицитами.

Рассмотрим ресурсно-балансовую модель государственного заказа на оказание специализированной, в том числе высокотехнологичной медицинской помощи в условиях круглосуточного стационара (СПС) по программе государственных гарантий (ПГГ). Математическая модель и алгоритмы оптимального распределения ресурсов на СПС здесь не рассматриваются.

Обозначим GF - общий объем финансовых средств на СПС, предусмотренных ПГГ; $\mathrm{JH}=\{\mathrm{k}\}-$ перечень видов (профилей) СПС, оплата которых предусмотрена госзаказом; $\mathrm{IM}=\{\mathrm{m}\}-$ множество всех медицинских организаций, которые могут оказывать СПС, то есть

«m Î IM, \$k ÎJH: WPkm > 0 (11)

где WPkm - производственный потенциал $\mathrm{m}$-ой МО по оказанию СПС k-го вида (профиля);

IG - множество MO, получивших госзаказ на оказание СПС, такое что IG Í IM.

Единицей распределения финансовых ресурсов при планировании государственного заказа на СПС является величина Qn - норматив возмещения финансовых затрат МО за один законченный случай оказания больному СПС n-го профиля. Профиль СПС будем сопоставлять с профилем койки.

Полагаем, что Qn - это средневзвешенная величина, рассчитанная с учетом всех случаев оказания СПС, относящихся к n-му профилю, то есть

$$
\mathrm{Qn}=(\mathrm{Qk} \times \mathrm{fk})(12)
$$

где $\mathrm{Qk}$ - нормативная стоимость оказания СПС k-го вида (профиля) одному больному, $\mathrm{fk}-$ частота оказания СПС k-го вида (профиля); суммирование выполняется по всем k ÎPn, где Pn множество случаев оказания СПС, относящихся к n-му профилю.

В свою очередь Qk может быть рассчитана как средняя стоимость стандартов медицинской помощи либо соответствующих клинико-

\footnotetext{
* Одним из направлений повышения эффективности деятельности медицинских организаций является передача отдельных функций на аутсорсинг. При этом происходит трансформация ряда внутренних бизнеспроцессов МО в соответствующие внешние бизнес-процессы.
} 
статистических групп (КСГ), относящихся к k-му виду СПС, то есть

$$
\mathrm{Qk}=(\mathrm{Qsi} \times \mathrm{fsi})(13)
$$

где Qsi - стоимость і-го стандарта или КСГ; fsi - частота его применения;

суммирование выполняется по всем i ÎMk, где $\mathrm{Mk}$ - множество стандартов (КСГ), относящихся к k-му виду (профилю) СПС.

Методы расчета Qsi мы здесь не рассматриваем. Полагаем, что Qsi могут быть рассчитаны для применения с территориальными коэффициентами удорожания условной единицы бюджетных услуг, устанавливаемыми Правительством РФ.

Сводный государственный заказ $\mathrm{G}$ на оказание СПС населению субъектов РФ для МО 3-го уровня и федеральных медицинских учреждений может быть представлен в виде кубической матрицы

$$
\mathrm{G}=\| \text { VPkmj } \|(14)
$$

где $\mathrm{VPkm}=\mathrm{VPkmj}-$ госзаказ $\mathrm{m}$-му MO на оказание k-ого вида (профиля) СПС, индекс j соответствует субъекту РФ, VPkmj - установленный объем для ј-го субъекта РФ по оказанию k-го вида СПС m-ым МО.

Государственный заказ $\mathrm{G}$ должен удовлетворять следующим условиям баланса:

a) для всех k ÎJH и m ÎIG должно быть VPkm < WPkm;

б) для всех k ÎJH и ј должно быть VPkmj < LPkj, где LPkj - «длина» листа ожидания k-ого вида СПС ј-го субъекта РФ;

в) $(\operatorname{VPkmj} \times \mathrm{Qk}(\mathrm{n}))=\mathrm{GF}$, где $\mathrm{Qk}(\mathrm{n})-$ установленный норматив стоимости возмещения за случай оказания k-го вида СПС, такой что для $\mathrm{k}$ ÎPn: $\mathrm{Qk}(\mathrm{n})=\mathrm{Qn}$; суммирование по индексу m выполняется по всем m IIIG.

Очевидно, что задача планирования специализированной больничной медицинской помощи GF $\{\mathrm{LPkj}\}\{\mathrm{WPkm}\}\{\mathrm{Qn}\}{ }^{\circledR}\|\mathrm{VPkmj}\|(15)$ относится к классу задач многокритериальной оптимизации. При этом следует отметить, что сегодня целевые функции, по которым осуществляется распределение ресурсов и оптимизация матрицы ||VPkmj || четко не определены ни в одном документе [16].

Интуитивно понятно, что одной из таких функций может быть, например, критерий

$\mathrm{VP}=\mathrm{VPkmj}{ }^{\circledR} \max (16)$

где VP - общее количество плановых больных, который может быть дифференцирован, в частности отдельно для детей и для взрослых.

Важнейшим показателем, отражающим доступность СПС для населения, является время ожидания $\mathrm{t}$, а критерием оптимизации $\mathrm{t}{ }^{\circledR}$ $\min$ - среднее время ожидания больными СПС от даты включения пациента в лист ожидания до даты фактического обращения в МO; здесь также возможна дифференциация критерия, при формировании программы госгарантий и распределению соответствующих субвенций на финансирование СПС по субъектам РФ:

1) пропорционально численности населения;

2) пропорционально «длине» LPkj листа ожидания субъекта РФ.

Очевидно, что подход (1) не вполне адекватен требованиям равнодоступности СПС, поскольку не учитывает фактических данных о потребности населения в СПС и поэтому может использоваться только тогда, когда отсутствуют полные и достоверные данные LPkj.

Возможно также применение комбинированных методов распределения объемов и субвенций, в которых указанные подходы используются совместно, например, по одним видам (профилям) СПС - пропорционально численности населения, по другим - по количеству больных, включенных в листы ожидания, или в которых учитываются и численность населения, и «длина» листа ожидания.

Заметим, что во всех случаях наиболее естественным является распределение объемов, при котором VPkj / VP = kv × LPkj / LP (17),

где $\mathrm{kv}<1.0$ - некоторый коэффициент, который может как зависеть от территории и(или) вида (профиля) СПС, так и быть от них независимым.

Формирование госзаказа для МО также может осуществляться на основе различных подходов. При этом в общем случае необходимо учитывать производственные мощности МО, их географическое расположение и транспортную доступность, потребности в соответствующих видах (профилях) СПС, в том числе территориально близкого населения и т.п.

Оценка производственного потенциала МО должна осуществляться комплексно с использованием дополняющих друг друга нормативного, статистического и аналитического методов планирования ресурсного обеспечения.

При использовании аналитического метода планирования ресурсного обеспечения МО необходимо руководствоваться взаимосвязями, возникающими между медицинскими работниками в рамках одного профиля медицинской помощи и профиля коечного фонда, а также 
между структурными подразделениями внутри медицинской организации и при работе с внешними контрагентами.

Оценочная характеристика результатов ресурсного обеспечения, полученных в ходе проведенного анализа, должна добавляться причинно-следственной диаграммой, акцентированной на тех ресурсах МО, использование которых может быть более эффективным [15].

\section{Библиографический список}

1. Kathleen Carey, James F Burgess, Jr., Gary J Young. Specialty and Full-Service Hospitals: A Comparative Cost Analysis Health Serv Res. 2008 Oct; 43(5 Pt 2): 1869-1887. doi: 10.1111/j.1475-6773.2008.00881. PMCID: PMC2654166.

2. Florien M. Kruse, Niek W. Stadhouders, Eddy M. Adang, Stef Groenewoud, Patrick P. T. Jeurissen Do private hospitals outperform public hospitals regarding efficiency, accessibility, and quality of care in the European Union? A literature review Int J Health Plann Manage. 2018 Apr-Jun; 33(2): e434-e453. Published online 2018 Mar 2. doi: 10.1002/hpm.2502 PMCID: PMC6033142.

3. May P, Garrido MM, Cassel JB, Morrison RS, Normand C. Using Length of Stay to Control for Unobserved Heterogeneity When Estimating Treatment Effect on Hospital Costs with Observational Data: Issues of Reliability, Robustness, and Usefulness. Health Serv Res. 2016 Oct; 51(5):2020-43. doi: 10.1111/1475-6773.12460. Epub 2016 Feb 21. PMID: 26898638.

4. Donna Lee Armaignac, Anshul Saxena, Muni Rubens, Carlos A.Valle, Lisa-Mae S. Williams, Emir Veledar, Louis T. Gidel. Impact of Telemedicine on Mortality, Length of Stay, and Cost Among Patients in Progressive Care Units: Experience From a Large Healthcare System. Crit Care Med. 2018 May; 46(5): 728-735. Published online 2018 Apr 13. doi: 10.1097/CCM.0000000000002994. PMCID: PMC5908255.

5. Hyunyoung Baek, Minsu Cho, Seok Kim, Hee Hwang, Minseok Song, Sooyoung Yoo Analysis of length of hospital stay using electronic health records: A statistical and data mining approach PLoS One. 2018; 13(4): e0195901. Published online 2018 Apr 13. doi: 10.1371/journal.pone.0195901 PMCID: PMC5898738

6. OECD (2017), Health at a Glance 2017: OECD Indicators, OECD Publishing, Paris. http://dx.doi.org/10.1787/ health_glance-2017-en.

7. McConnell KJ, Chang AM, Maddox TM, Wholey DR, Lindrooth RC. An exploration of management practices in hospitals. Healthcare. 2014;2(2): 121-9.

8. Drotz E, Poksinska B. Lean in healthcare from employees’ perspectives. J Health Organ Manag. 2014;28(2):177-95.

9. Timmons $S$, Coffey $F$, Vezyridis $P$. Implementing lean methods in the emergency department: the role of professions and professional status. J Health Organ Manag. 2014;28(2):214-28.

10. Martin AB, Hartman $M$, Whittle L, Catlin A. National health spending in 2012: Rate of health spending growth remained low for the fourth consecutive year. Health Affairs. 2014; 33(1):67-77. https://doi.org/10.1377/ hlthaff.2013.1254 PMID: 24395937.

11. Качкова О.Е., Кришталева Т.И. Методика оценки и расчета показателей результативности деятельности медицинских организаций//Актуальные проблемы социально-экономического развития России. 2020. № 4. С.30-36.

12. Хальфин Р. А., Орлов С.А., Мадьянова В.В., Столбов А.П., Качкова О.Е. Стандартизация подходов к оценке лечебно-диагностического процесса и мониторинг использования ресурсов медицинской организации в условиях стационара//Проблемы стандартизации в здравоохранении. 2020. № 1-2. С.3-10

13. Хальфин Р.А., Орлов С.А., МадьяноваВ.В., СтолбовА.П., Качкова О.Е. Современные подходы к оценке эффективности использования ресурсов здравоохранения (обзор)// Проблемы стандартизации в здравоохранении. 2020. № 3-4. С.3-12.

14. Хальфин Р.А., Орлов С.А., Мадьянова В.В., Столбов А. П., Качкова О.Е. О методах оценки и расчета производственного потенциала медицинской организации, оказывающей специализированную медицинскую помощь в условиях круглосуточного стационара//Проблемы социальной гигиены, здравоохранения и истории медицины. 2020. Т. 28.№ 5. С. 964-969.

15. Хальфин Р.А., Муравьев Д. Н., КакоринаЕ.П., Розалиева Ю. Ю, Кришталева Т.И. Анализ результатов внедрения подушевой оплаты с элементами фондодержания в Российской Федерации//Проблемы социальной гигиены, здравоохранения и истории медицины. 2020. Т. 28. № 6. С. 1333-1338

16. Хальфин Р.А., ОрловС.А., МадьяноваВ.В., СтолбовА.П., КачковаО.Е.Стандартизированный алгоритм медико-экономической оценки потенциала экспорта медицинских услуг/Проблемы стандартизации в здравоохранении. 2019. № 11-12. С.9-14. 